\title{
Kin selection and reproductive value in social mammals
}

\author{
Masaru Hasegawa $^{1}$ - Nobuyuki Kutsukake ${ }^{1}$ (D)
}

Received: 17 March 2018 / Accepted: 27 January 2019 / Published online: 15 February 2019

(c) The Author(s) 2019, corrected publication 2019

\begin{abstract}
Empirical studies have tested and confirmed a positive relationship between relatedness and the degree of altruistic behavior, as predicted by kin selection theory. In contrast, researchers have studied the relationship between reproductive value - the extent to which an individual of a given "state" contributes to future generations - and the degree of altruistic behavior far less frequently, although reproductive value should be considered when testing kin selection. Here, we reviewed empirical studies examining such relationships in social mammals. Overall, $>50 \%$ of these studies demonstrated that the degree of altruistic behavior (1) decreases with the reproductive value of the actor, and (2) increases with the reproductive value of the recipient, supporting the importance of reproductive value in altruistic behavior. However, these studies cannot rule out all other explanations than kin selection. Few empirical studies have adopted a stronger test that uses the product of relatedness and reproductive value (i.e., their two-way interaction in a linear model). Such tests should be particularly useful when studying long-lived vertebrates, for which the direct estimation of fitness benefits and costs is impractical. To achieve a better understanding of the evolution of kin-directed behaviors, future studies should use the product of relatedness and reproductive value.
\end{abstract}

Keywords Altruism $\cdot$ Kin selection $\cdot$ Kinship $\cdot$ Social behavior $\cdot$ Reproductive value

\section{Introduction}

"The extent of altruism should be a function of the product of the receiver's reproductive value and his degree of relatedness to the altruist." (Milinski 1978).

It has been more than 35 years since several researchers (e.g., Emlen 1973; Milinski 1978; Altmann 1979) advocated considering reproductive value as well as relatedness in empirical tests of kin selection theory (Hamilton 1964). Reproductive value is a standard measure of the expected contribution of an individual in a given state to the future population (Fisher 1930; Taylor 1990; Frank 1998; Houston and McNamara 1999). In other words, reproductive

Electronic supplementary material The online version of this article (https://doi.org/10.1007/s10164-019-00586-6) contains supplementary material, which is available to authorized users.

Nobuyuki Kutsukake

kutsu@soken.ac.jp

1 Department of Evolutionary Studies of Biosystems, Sokendai (The Graduate University for Advanced Studies), Shonan Village, Hayama, Kanagawa 240-0193, Japan value represents the relative contribution of a state (e.g., an individual at a particular age) to descendants far into the future, i.e., the number of descendants left by an individual in a particular state relative to those left by an individual in a reference state (Houston and McNamara 1999; see also Appendices 1 and 2 for calculations). Reproductive value can be calculated in any state-structured matrix as the dominant left eigenvector of a given population matrix (Caswell 1989; Grafen 2006), though it is most often utilized in an age-structured matrix (i.e., a Leslie matrix), as was first advocated by Fisher (1930). For example, as shown in Fig. 1, individuals in different age classes (where age class is a state variable) markedly differ in their expected contributions to the future population (e.g., the contribution of 2-year-old yellow-bellied marmot females to future descendants is fourfold that of 1-year-old females; Fig. 1). This is because states differ in their transition rates to other states (i.e., survival and fecundity in an age-structured population; see Appendix 1 for their contributions to reproductive values).

Reproductive value has a huge impact on the propagation of shared genes when kin-directed behavior occurs, so it should be considered in empirical tests of kin selection. Milinski (1978) succinctly summarized core predictions incorporating reproductive value as follows. First, 


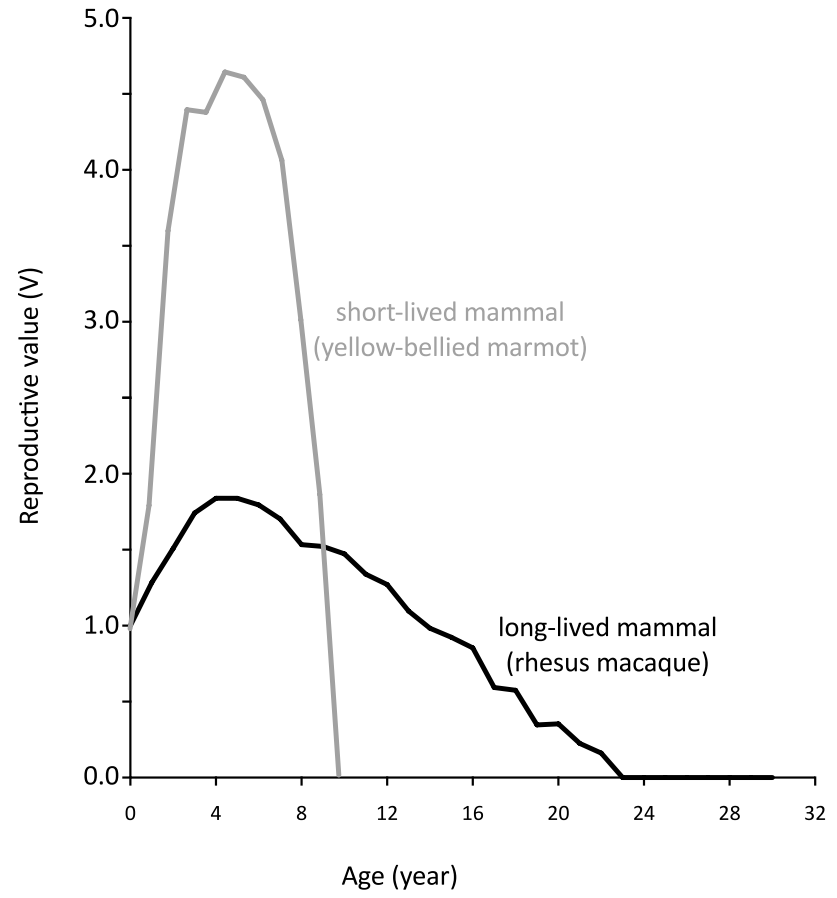

Fig. 1 Two examples of reproductive values of mammals based on the Leslie matrix: the female rhesus macaque as a representative long-lived mammal (data from Table A1 in the Appendix of Hernández-Pacheco et al. 2013), and the female yellow-bellied marmot, Marmota flaviventris, as a representative short-lived mammal (data from Schwartz et al. 1998)

the degree of altruism (i.e., social interactions that benefit recipients at the expense of the fitness of the actor; see West et al. 2007) among relatives should decrease with the actor's reproductive value. Second, the degree of altruism among relatives should increase with the recipient's reproductive value. Third, the extent of altruism should be positively related to the product of the receiver's reproductive value and his degree of relatedness to the altruist (Milinski 1978). These predictions, however, have received limited attention in empirical studies, due in part to a lack of understanding of the advantage or the theoretical background, or to the scattered empirical evidence, all of which prevent empirical researchers from incorporating reproductive value into their studies of kin selection.

The purpose of the current paper is to advocate for the use of reproductive value in empirical studies of kin selection. To this end, we first explain the advantage of using reproductive value in kin selection studies and the theoretical perspectives of these predictions. Then we review empirical studies examining the three predictions noted above (and their derivatives) to determine general patterns and future prospects. We focus on social interactions of mammal societies, which should be particularly suited to the application of the predictions. In mammals that form "individualized societies" (sensu de Waal and Tyack 2003; also see Bergman and Beehner 2015), a longitudinally stable group contains long-term and multigenerational relationships (de Waal and Tyack 2003), resulting in variation in relatedness among dyads and according to other individual attributes (e.g., age, dominance; Kutsukake 2009). Group members have repeated opportunities to interact with and recognize other group members, and are predicted to employ social tactics that are fine-tuned to their own and group members' attributes as well as to the relatedness to the partner. Considering reproductive value permits unique predictions that are useful for testing kin selection explanations without being confounded by alternative explanations of (seemingly) altruistic behaviors such as reciprocity (West et al. 2007; Bergmüller et al. 2007), as we explain below. Although we mainly focus on mammals, the same logic based on reproductive value can be applied to other taxa.

\section{Tests of Hamilton's rule are impractical in empirical studies}

The three predictions above are still useful for studying kin selection today because the direct estimation of fitness benefits (of recipient $b$ ) and costs (of actor $c$ ) in Hamilton's rule (i.e., $r b-c>0$, where $r$ denotes relatedness; Hamilton 1964) is impractical, particularly in long-lived animals (e.g., mammals). In these animals, fitness benefits and costs cannot be measured (e.g., a grooming event may have a subtle effect on the actor and recipient fitness), which explains why only a limited number of empirical studies have tested Hamilton's rule (e.g., see Bourke 2014, in which no mammal studies are included). Immediate effects of altruistic behavior such as increases in survival or fecundity can sometimes be measured in these species, but measuring effects of kindirected behavior on survival or fecundity is not enough to apply Hamilton's rule. For example, one may assume that a $30 \%$ increase in offspring production of the recipients offsets a $<7.5 \%$ decrease in offspring production of the altruist when their relatedness $r=0.25$. However, this is not true. When a recipient produces lower quality offspring than an altruist, the altruist should cease their altruistic behavior toward the recipient (also see Appendix 2). In other words, $b$ and $c$ measure the long-term contribution to the descendant rather than the immediate increase/decrease in survival, fecundity, or lifetime reproductive success. Even the number of grandchildren or great grandchildren will often be inadequate (Houston and McNamara 1999). Therefore, we should take account of long-term fitness using the reproductive value (i.e., benefits/costs in terms of descendants far into the future cannot be estimated without using reproductive value - see the next section for a detailed explanation; also 
note that, in some cases, immediate effects cannot be directly compared, e.g., increased fecundity of the recipient at the expense of the survival of the actor).

Clearly, examining nepotism (i.e., the positive relationship between $r$ and social behaviors) is not enough to study social behaviors in the context of kin selection, even in combination with the immediate effect of the behaviors on fitness components, as we exemplified above. Considering reproductive value would advance our understanding of kindirected behavior and its function in wild animals. So, how does the consideration of reproductive value increase our understanding of social behaviors? In this present work, we focused on the theoretical perspectives of reproductive value in the context of kin selection, particularly its mathematical equivalence to Hamilton's rule and how it enhances the tractability of Hamilton's rule (even if fitness benefits/costs or immediate effects cannot be estimated).

\section{Theoretical perspective}

From a theoretical perspective, the above predictions match the derived form of Hamilton's rule in a state-structured population, $r b^{\prime} V_{\text {recipient }}-c^{\prime} V_{\text {actor }}>0$ (Taylor and Frank 1996; Frank 1998), where $V_{\text {actor }}$ and $V_{\text {recipient }}$ denote the reproductive values for an actor and a recipient in forthcoming (not the current) states; $b^{\prime}$ and $c^{\prime}$ represent the benefits and costs, measured as the immediate changes in survival or reproduction. We use $b^{\prime}$ and $c^{\prime}$ here to distinguish this from Hamilton's original rule (note that Hamilton's rule incorporates all fitness components, i.e., offspring production, the timing of reproduction, and the state of offspring were also considered; see Oli 2003). In a state-structured population, fitness benefit $b$ and cost $c$ in Hamilton's original rule can be regarded as $b^{\prime} V_{\text {recipient }}$ and $c^{\prime} V_{\text {actor }}$, respectively. More technically, these represent changes in the transition rate from a given state to the forthcoming states after an actor's behavior. For example, in species that live for two years, the transition can consist of annual survivorship (e.g., infants to yearlings, and yearlings to 2-year-olds) and infant production by yearlings or 2-year-olds (Frank 1998). Thus, $b^{\prime} V_{\text {recipient }}$ and $c^{\prime} V_{\text {actor }}$ represent increased and decreased contributions to descendants far into the future for the recipient and actor, respectively, by the focal behavior. For example, when an actor's behavior increases the yearly survivorship of any mature recipients by $10 \%$ (i.e., $b^{\prime}=0.10$ ), the behavior provides the actor with a fitness benefit in proportion to the recipients' reproductive value (e.g., if the reproductive value for age 4, 5, $6=0.5,1$, 2 , respectively, then the fitness benefit by supporting ages 3,4 , and 5 will be $0.05,0.10,0.20$, respectively). Reproductive value can consider three fitness correlates-in addition to the total number of offspring produced (a well-known fitness correlate), reproductive value can also consider the timing of reproduction and the state of offspring (Houston and McNamara 1999). State can be any variable, including sex, size, stage, dominance, body condition, and age. Reproductive values based on age can be calculated using the Leslie matrix (or the Lefkovitch matrix for reproductive value based on other categorical state variables).

Assuming that $b^{\prime}$ and $c^{\prime}$ are unbiased among candidate recipients and actors, Taylor and Frank's inequality, $r b^{\prime} V_{\text {recipient }}-c^{\prime} V_{\text {actor }}>0$, yields nepotism (i.e., the degree of altruism should increase with the relatedness between actor and recipient) and the above predictions of reproductive value. That is, the degree of altruism among relatives should decrease with the actor's reproductive value (i.e., prediction A) and increase with the recipient's reproductive value (i.e., prediction B). By using $r V_{\text {recipient }}$ as a unit, we can derive a prediction that the extent of altruism increases with the product of the receiver's reproductive value and its degree of relatedness to the altruist (i.e., prediction C).

\section{Empirical studies}

We provide a review of empirical studies testing the first two simple predictions that the degree of altruism among relatives should (1) decrease with the actor's reproductive value (Fig. 2a) and (2) increase with the recipient's reproductive value (Fig. 2b). The derivative of these two predictions is that individuals with lower expected reproductive values should invest in individuals with higher expected reproductive values (Fig. 2c); thus, we also provide an empirical test of the prediction combining these two simple predictions ("prediction A\&B" hereafter). Lastly, in addition to these predictions that focus solely on reproductive value, we present an empirical study investigating the powerful prediction (prediction C) that the extent of altruism can be explained by the product of the receiver's reproductive value and their degree of relatedness to the altruist (Milinski 1978).

\section{Literature survey}

For this purpose, we gathered literature focusing on reproductive value in the context of social behavior. Articles were searched for using Google Scholar with the keywords "reproductive value" AND mammal AND behavior OR behaviour. We collected all of the matching papers published in scientific journals before 31st December 2015 (total 152 studies; Table S1 in the Electronic supplementary material, ESM). When gathering information, we did not distinguish among methodologies of measuring reproductive value (e.g., projection matrix or other methods) or relatedness (e.g., pedigree or genetic marker).

Because reproductive value can be predicted from state variables (e.g., reproductive value increases until 
(a)

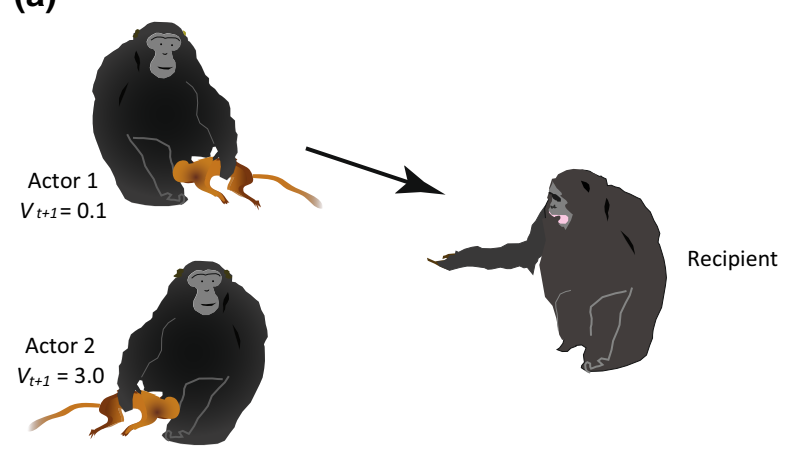

(b)

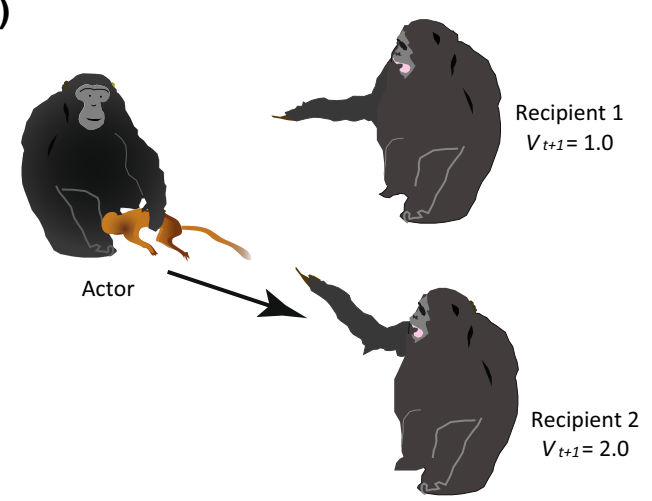

(c)

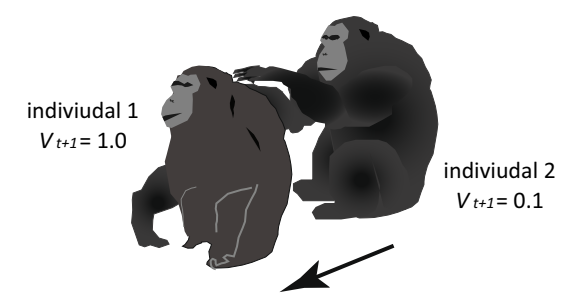

Fig. 2 Simple Predictions of altruistic behavior based on reproductive value among kin individuals given the same relatedness $r$. Each individual with a given state at time $t$ is shown with a given expected reproductive value at the next time period $\left(V_{t+1}\right)$, and solid arrows indicate the predicted main directions of the altruistic behavior. a

maturation and then decreases with age; see Appendix 3 for the background logic), we included all studies that examine the pattern between reproductive value (or its indirect measures, such as age, sex, and so on) and social interactions, including parental investment. For example, in life history studies, terminal investment is a well-known phenomenon in which older individuals (often mothers) with low expected reproductive values (i.e., low "residual reproductive value;" Pianka and Parker 1975; see also Morris 1986) invest more in current reproduction than younger individuals do. This pattern can be tested without directly measuring reproductive value; indeed, most studies have tested this pattern using age, which is tightly linked to reproductive values and is easier to estimate than reproductive value. We judged whether the prediction was supported or not based on the authors' conclusion, founded on statistical analyses. Because the use of such indirect measures leads to some uncertainty compared with directly estimating the reproductive value, we also focused on patterns among studies that directly estimated reproductive values.

Our database included studies of social interactions and parental investment that might accompany behavioral components. We excluded data on nonsocial behaviors (e.g., foraging, mating, or personality traits; note that we included
Case in which there are two potential actors that could benefit a recipient. b Case in which an actor can benefit one of two potential recipients. In $\mathbf{a}$ and $\mathbf{b}$, an example context is meat sharing in chimpanzees. c Case in which two related individuals will be either actor or recipient. In c, an example context is grooming in chimpanzees

parent-offspring interactions because, theoretically, social interactions between parent and offspring and those between other relatives are not different).

Although this type of literature review often accompanies a quantitative meta-analytic approach using effect size, we avoided such an approach because of our highly heterogeneous dataset. For example, most studies have not used reproductive value itself; they have used state variables such as age or sex instead (note that, in empirical studies, some states are known to have higher reproductive values than others, e.g., older individuals have lower reproductive values after maturation; see Appendix 3). For this reason, effect size is difficult to interpret. Even if reproductive value increases with age until maturation (and decreases thereafter), the exact increase or decrease in reproductive value per age depends on each age class as well as the species' life history (Fig. 1), so effect size based on age or other state variables has little meaning when used to infer the quantitative effect of reproductive value. Moreover, predictions based on reproductive value are highly variable, i.e., some analyses predict simple linear relationships between reproductive value (or the state variable) and social behaviors, while others predict specific patterns of behavior (e.g., the existence of "youngest ascendancy," as we explain in the discussion of prediction B later in the paper). Therefore, we 
adopted a classic (or narrative) literature review in which we summarized the overall pattern. This type of literature review based on the overall pattern is normally less effective at identifying a general pattern than a quantitative metaanalysis (e.g., see Borenstein et al. 2009 for a recent review). Thus, our review may have underestimated rather than overestimated the overall effect of reproductive value. Still, we think that this approach is a good starting point for reviewing the currently scattered information on reproductive value and stimulating a more systematic review in the future.

\section{Overall pattern}

Overall, $>50 \%$ of empirical studies have supported these predictions (Table 1; also see Table S1 in the ESM for detailed information). Although those studies have mostly been correlational, experimental studies have also been performed (Schwanz 2008a, b; see also Nehring et al. 2012 for a similar experiment in social insects). Most studies have substituted age for reproductive value due to the predictable relationship between the two (Fig. 1); only a few studies have calculated reproductive value (Table 1; see Table S1 in the ESM for detailed information). Thus, most studies are based on the assumption that reproductive values change in a predictable manner in age-structured populations, and its age-related pattern depends on the species considered, so caution must be applied during

Table 1 Summary of a review of the literature supporting predictions

\begin{tabular}{llll}
\hline & Overall & \multicolumn{2}{l}{ Sorted by state variable } \\
\cline { 3 - 4 } & & Age alone & Others \\
\hline Overall pattern & & & \\
Prediction A & $51 \%(42 / 83)$ & $46 \%(32 / 69)$ & $71 \%(10 / 14)$ \\
Prediction B & $74 \%(42 / 57)$ & $50 \%(4 / 8)$ & $78 \%(38 / 49)$ \\
Prediction A\&B & $91 \%(10 / 11)$ & $100 \%(9 / 9)$ & $50 \%(1 / 2)$ \\
Prediction C & $0 \%(0 / 1)$ & $0 \%(0 / 1)$ & $-(0 / 0)$ \\
Total & $62 \%(94 / 152)$ & $52 \%(45 / 87)$ & $75 \%(49 / 65)$ \\
Studies using state variables & & \\
Prediction A & $50 \%(38 / 76)$ & $45 \%(28 / 62)$ & $71 \%(10 / 14)$ \\
Prediction B & $76 \%(41 / 54)$ & $60 \%(3 / 5)$ & $78 \%(38 / 49)$ \\
Prediction A\&B & $90 \%(9 / 10)$ & $100 \%(8 / 8)$ & $50 \%(1 / 2)$ \\
Prediction C & $-(0 / 0)$ & $-(0 / 0)$ & $-(0 / 0)$ \\
Total & $63 \%(88 / 140)$ & $52 \%(39 / 75)$ & $75 \%(49 / 65)$ \\
Studies calculating reproductive values & & \\
Prediction A & $57 \%(4 / 7)$ & $57 \%(4 / 7)$ & $-(0 / 0)$ \\
Prediction B & $33 \%(1 / 3)$ & $33 \%(1 / 3)$ & $-(0 / 0)$ \\
Prediction A\&B & $100 \%(1 / 1)$ & $100 \%(1 / 1)$ & $-(0 / 0)$ \\
Prediction C & $0 \%(0 / 1)$ & $0 \%(0 / 1)$ & $-(0 / 0)$ \\
Total & $50 \%(6 / 12)$ & $50 \%(6 / 12)$ & $-(0 / 0)$ \\
\hline
\end{tabular}

See main text for a detailed explanation of each prediction interpretation. Only a fraction of the reported studies used other state variables (e.g., size, rank, condition) when testing the predictions, except for prediction $\mathrm{B}$, in which sex rather than age was used as a predominant state variable (Table 1). Unfortunately, when investigating social interactions, no study has calculated reproductive value based on state variables other than age (Table 1).

Although some studies have focused on reproductive effort in which a behavioral component is not ascertained (e.g., milk provision/intake), studies with clear behavioral components (e.g., defensive behavior, grooming, aggression, and so on) have demonstrated similar patterns to those obtained in all studies (Tables 1,2). Thus, again, the majority (66\%) of the studies found support for the importance of reproductive value (Table 2). Most of these studies focused on the mother-offspring or sibling relationship (Table 2), but similar findings (i.e., support for the prediction based on reproductive value) have been obtained in studies on grandmothers, aunts (Fairbanks 1988), and more distant relatives (Borries et al. 1994). We provide a short summary of the literature review, a detailed explanation, and some examples for each prediction below.

\section{Prediction A: The frequency/intensity of investment between related individuals should be negatively related to the reproductive values of the actors}

In the 83 studies that we listed, $50.6 \%$ of them showed positive results, although only a few estimated reproductive value (Table 1). Among all 83 studies, age was the most frequently used state variable, and support for this state variable was slightly below $50 \%$ (46.4\%; Table 1 ). The majority of the studies supported the prediction when we focused solely on studies with clear behaviors (i.e., focused on a limited number of studies; see Table 2). An alternative explanation for this pattern is that animals effectively adjust their investment towards kin members through behavioral components rather than other components (e.g., purely physiological maternal investment). The effect size of age-based reproductive value remains to be clarified in the future.

In prediction $\mathrm{A}$, actors who are expected to have low future reproductive values should invest more in kin members than should those with high reproductive values. Since the first application in Hrdy and Hrdy (1976), several studies have supported this prediction. In female Hanuman langurs, Presbytis entellus, Hrdy and Hrdy (1976) found that older females had a low reproductive value and in fact behaved altruistically, i.e., were subordinate and spent more time defending the troop, while young females had a high reproductive value and hence behaved selfishly, i.e., were more dominant and defended the troop less (also see Croft et al. 2015 for post-reproductive lifespan). 
Table 2 Summary of a review of the literature supporting predictions when confined to studies of clear behavior

\begin{tabular}{|c|c|c|c|c|c|}
\hline & \multirow[t]{3}{*}{ Overall } & \multicolumn{4}{|l|}{ Sorted by } \\
\hline & & \multicolumn{2}{|l|}{ State variable } & \multicolumn{2}{|l|}{ Participants } \\
\hline & & Age alone & Others & Close relatives & Others \\
\hline \multicolumn{6}{|l|}{ Overall pattern } \\
\hline Prediction A & $62 \%(24 / 39)$ & $59 \%(20 / 34)$ & $80 \%(4 / 5)$ & $54 \%(15 / 28)$ & $82 \%(9 / 11)$ \\
\hline Prediction B & $63 \%(12 / 19)$ & $43 \%(3 / 7)$ & $75 \%(9 / 12)$ & $63 \%(10 / 16)$ & $67 \%(2 / 3)$ \\
\hline Prediction A\&B & $100 \%(8 / 8)$ & $100 \%(7 / 7)$ & $100 \%(1 / 1)$ & $100 \%(5 / 5)$ & $100 \%(3 / 3)$ \\
\hline Prediction C & $0 \%(0 / 1)$ & $0 \%(0 / 1)$ & $-(0 / 0)$ & $-(0 / 0)$ & $0 \%(0 / 1)$ \\
\hline Total & $66 \%(44 / 67)$ & $61 \%(30 / 49)$ & $78 \%(14 / 18)$ & $61 \%(30 / 49)$ & $78 \%(14 / 18)$ \\
\hline \multicolumn{6}{|c|}{ Studies using state variables } \\
\hline Prediction A & $63 \%(22 / 35)$ & $60 \%(18 / 30)$ & $80 \%(4 / 5)$ & $54 \%(14 / 26)$ & $89 \%(8 / 9)$ \\
\hline Prediction B & $69 \%(11 / 16)$ & $50 \%(2 / 4)$ & $75 \%(9 / 12)$ & $64 \%(9 / 14)$ & $100 \%(2 / 2)$ \\
\hline Prediction A\&B & $100 \%(7 / 7)$ & $100 \%(6 / 6)$ & $100 \%(1 / 1)$ & $100 \%(4 / 4)$ & $100 \%(3 / 3)$ \\
\hline Prediction C & $-(0 / 0)$ & $-(0 / 0)$ & $-(0 / 0)$ & $-(0 / 0)$ & $-(0 / 0)$ \\
\hline Total & $69 \%(40 / 58)$ & $65 \%(26 / 40)$ & $78 \%(14 / 18)$ & $61 \%(27 / 44)$ & $93 \%(13 / 14)$ \\
\hline \multicolumn{6}{|c|}{ Studies calculating reproductive values } \\
\hline Prediction A & $50 \%(2 / 4)$ & $50 \%(2 / 4)$ & $-(0 / 0)$ & $50 \%(1 / 2)$ & $50 \%(1 / 2)$ \\
\hline Prediction B & $33 \%(1 / 3)$ & $33 \%(1 / 3)$ & $-(0 / 0)$ & $50 \%(1 / 2)$ & $0 \%(0 / 1)$ \\
\hline Prediction A\&B & $100 \%(1 / 1)$ & $100 \%(1 / 1)$ & $-(0 / 0)$ & $100 \%(1 / 1)$ & $-(0 / 0)$ \\
\hline Prediction $\mathrm{C}$ & $0 \%(0 / 1)$ & $0 \%(0 / 1)$ & $-(0 / 0)$ & $-(-/-)$ & $0 \%(0 / 1)$ \\
\hline Total & $44 \%(4 / 9)$ & $44 \%(4 / 9)$ & $-(0 / 0)$ & $60 \%(3 / 5)$ & $25 \%(1 / 4)$ \\
\hline
\end{tabular}

See main text for a detailed explanation of each prediction

Note that all studies without clear behaviors focused on close relatives, i.e., parent-offspring or sibling relationships (see Table S1 of the ESM)
Likewise, other empirical evidence supports the idea that older individuals (often mothers) with low expected reproductive values (i.e., low "residual reproductive value;" Pianka and Parker 1975; see also Morris 1986) invested more in current reproduction ("terminal investment;" Clutton-Brock 1991; note that the state variable 'age' was often used as a substitute for reproductive value due to the predictable link between age and reproductive value; also see the next section for the potential problems this might cause). For example, Descamps et al. (2007) showed that female red squirrels, Sciurus vulgaris, invested more in their offspring as they aged (in this case, the propensity to bequeath their territory to juveniles increased with female age). In addition to these correlational studies, experimental studies have also been performed. When the residual reproductive value was reduced by parasite infection in deer mice, Peromyscus maniculatus, females increased their current reproductive output, producing heavier offspring at birth compared with those in a control group (Schwanz 2008a, b).
Prediction B: The frequency/intensity of investment between related individuals should be positively related to the reproductive values of the recipients

In the 57 studies that we listed, the majority (73.7\%) of them showed positive results, although only three estimated reproductive value (one of the three supported the prediction). In contrast to other predictions in which age was used as a major state variable, most studies used sex as a state variable in this prediction (35 studies, of which 28 supported the prediction). Only a few studies used age as a state variable (Table 1). Although most studies focused on sex allocation, in which behavioral components are not clearly mentioned, some studies focused on specific types of behaviors (see Table 2).

In prediction $\mathrm{B}$, actors should prefer kin individuals with a high reproductive value over others of the same relatedness. Offspring age has been the focus of previous studies, as reproductive value should change predictably with age: older offspring have a higher reproductive value than that of younger ones until maturation, and vice versa after maturation. Schulman and Chapais (1980) provided a simple application. They proposed that the youngest ascendancy, in which the youngest daughter obtained the highest rank among sisters in cercopithecine primates (and the spotted 
hyena Crocuta crocuta; Holekamp and Smale 1993), could be explained by maternal support for the youngest daughter over older siblings, because the youngest daughters would have the highest reproductive value when they matured.

Similarly, the reproductive value of offspring depends on their sex (Trivers and Willard 1973; Milinski 1978; Leimar 1996). As predicted based on the primary sex ratio (Leimar 1996; Komdeur 2012; Schindler et al. 2015), mothers should allocate more postnatal investment to the sex with the highest expected reproductive value, depending on their own state (i.e., sex-biased parental investment; note that the sex with the highest reproductive value depends on the life history of the species considered). For example, in the bank vole (Clethrionomys glareolus), mothers allocate more resources to daughters than sons, which is predicted because a larger body size is more important for female reproductive success in this species (Koskela et al. 2009). Although these examples focus on the reproductive value of single offspring, recipients need not be single offspring-they can be groups (e.g., litter size, in which the reproductive value of the litter equals the sum of the reproductive values of the individuals; Montgomerie and Weatherhead 1988; e.g., the bank vole; Koskela et al. 2000).

\section{Prediction A\&B}

All of the studies except for one showed positive results, although this category was less well studied than separate analyses of predictions A and B (Table 1). A comparison of dyads would control for some potential confounding factors such as relatedness (e.g., mother and daughter have the same relatedness to each other). This might contribute to the high support for this prediction, though the small sample size might have also affected the results. Most of the studies (70\%) accompany specific types of behavior (Table 2).

Individuals with lower expected reproductive values should invest in individuals with higher expected reproductive values, rather than vice versa. For example, Kurland (1977) showed that older Japanese macaques, Macaca fuscata (and thus individuals with smaller reproductive values), groomed younger counterparts, rather than vice versa, among siblings and parents-offspring. These analyses that focus on which of the participants act altruistically represent special cases in which actors and recipients have an investment opportunity. In other cases, opportunities for altruistic acts would differ among the participants (e.g., parental investment). Relative reproductive values affect the actors' behavior (reviewed in Lindström 1999 for parental investment), and rank reversal between parent and offspring is one such example. In the savannah baboon, Papio cynocephalus, Combes and Altmann (2001) found that rank reversal occurred when a daughter's reproductive value became higher than that of the mother.

\section{Prediction C: The extent of altruism can be explained by the product of the receiver's reproductive value and their degree of relatedness to the altruist}

To date, only one empirical study has examined this relationship (see Tables 1 and 2; Van Horn et al. 2007), which corresponds to only $0.7 \%$ of all studies included (i.e., 1/152). Van Horn et al. (2007) studied group choice by female savannah baboons (Papio cynocephalus) during group fission events. In their study, relatedness played a significant role in female group choice in some events but not in others. When the reproductive value was considered, the results did not change qualitatively, indicating that the product of reproductive value and relatedness did not increase the predictability of social behavior. As Van Horn et al. (2007) wrote, it is unclear if females could not assess the reproductive values of others or if kin were of value to females through their direct benefits rather than their indirect benefits. Another possibility is that a reproductive value based on a specific time point (i.e., at group division) might not predict longterm association after group division. It would therefore be premature to propose a general pattern for this prediction.

\section{Advantages of testing prediction C}

As we demonstrated above, empirical studies that have tested and support predictions A and B are now accumulating, but few have focused on the product of reproductive value and relatedness. We suggest that it is critical to consider this product not only when testing the role of reproductive value but also when testing the kin selection explanation in empirical studies. We explain each of these in turn.

\section{Advantage 1: Widening the applicability of Hamilton's rule}

The product of reproductive value and relatedness provides a better perspective on social behavior than do separate analyses of each factor. Imagine a case in which an actor has to choose a recipient from among a full sibling, a half-sibling, or non-kin with differing future reproductive values $(1,3,5$, respectively; Fig. 3). Assuming constant $b^{\prime}$ and $c^{\prime}$ values at the social interaction, neither the individual with the highest reproductive value (non-kin) nor the one with the highest relatedness (full sibling) should be chosen. Rather, we can predict that the half-sibling with the highest $r V$ value will be chosen. The same prediction holds unless $b^{\prime} / c^{\prime}$ is at least 1.5-fold higher for the first than for the second individual. Thus, studying $r V$ would greatly widen the applicability of Hamilton's rule. 


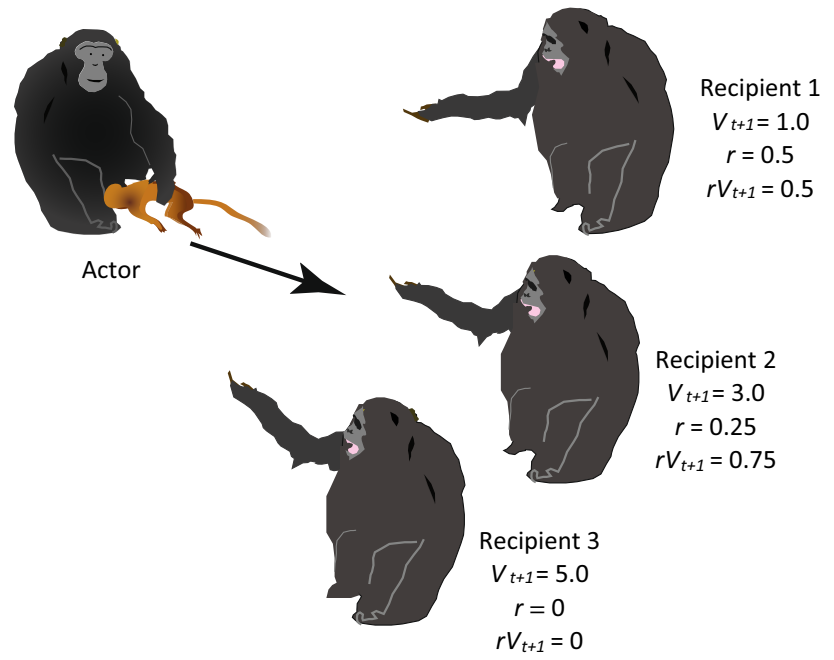

Fig. 3 The predicted effects of relatedness and reproductive value on altruistic behavior, exemplified by meat sharing in chimpanzees. An actor chooses from among a given set of potential recipients. Each individual with a given state at time $t$ has given expected reproductive values at the next time period $\left(V_{t+1}\right)$. The solid arrows indicate the predicted main directions of the altruistic behavior (see main text for more detailed information)

A rational but rarely conducted approach to testing this prediction is to use linear models, as done in nepotism studies (reviewed in Smith 2014) or in studies of reproductive value, as we reviewed here (Tables 1,2). By including the product of reproductive value and relatedness, i.e., $r V$, as an independent variable, one can test whether the extent of altruism should be a function of the product of the receiver's reproductive value and his/her degree of relatedness to the altruist.

Rather than including the product, a better approach would be to include the interaction term between $r$ and $V_{\text {recipient }}$ (i.e., $r V_{\text {recipient }}$ ) as well as each main effect, so that predictions from the kin selection theory could be tested in linear models with the degree (e.g., frequency) of altruistic behavior as a response variable (Fig. 4a, b). This approach is necessary to study whether the product increases the predictability of the focal behavior, as predicted by theory. In addition to the influence of these terms, altruistic behavior on the part of an actor should also be examined in linear models (i.e., it should decrease monotonically with $V_{\text {actor }}$; Fig. 4c). Because there are ample studies investigating nepotism (Smith 2014) and reproductive value (this study), the lack of empirical studies that use the interaction term together with the main terms would be simply due to ignorance or underestimation of its importance (see Smith 2014 for a detailed explanation of each method). This point will be explained in the next section.
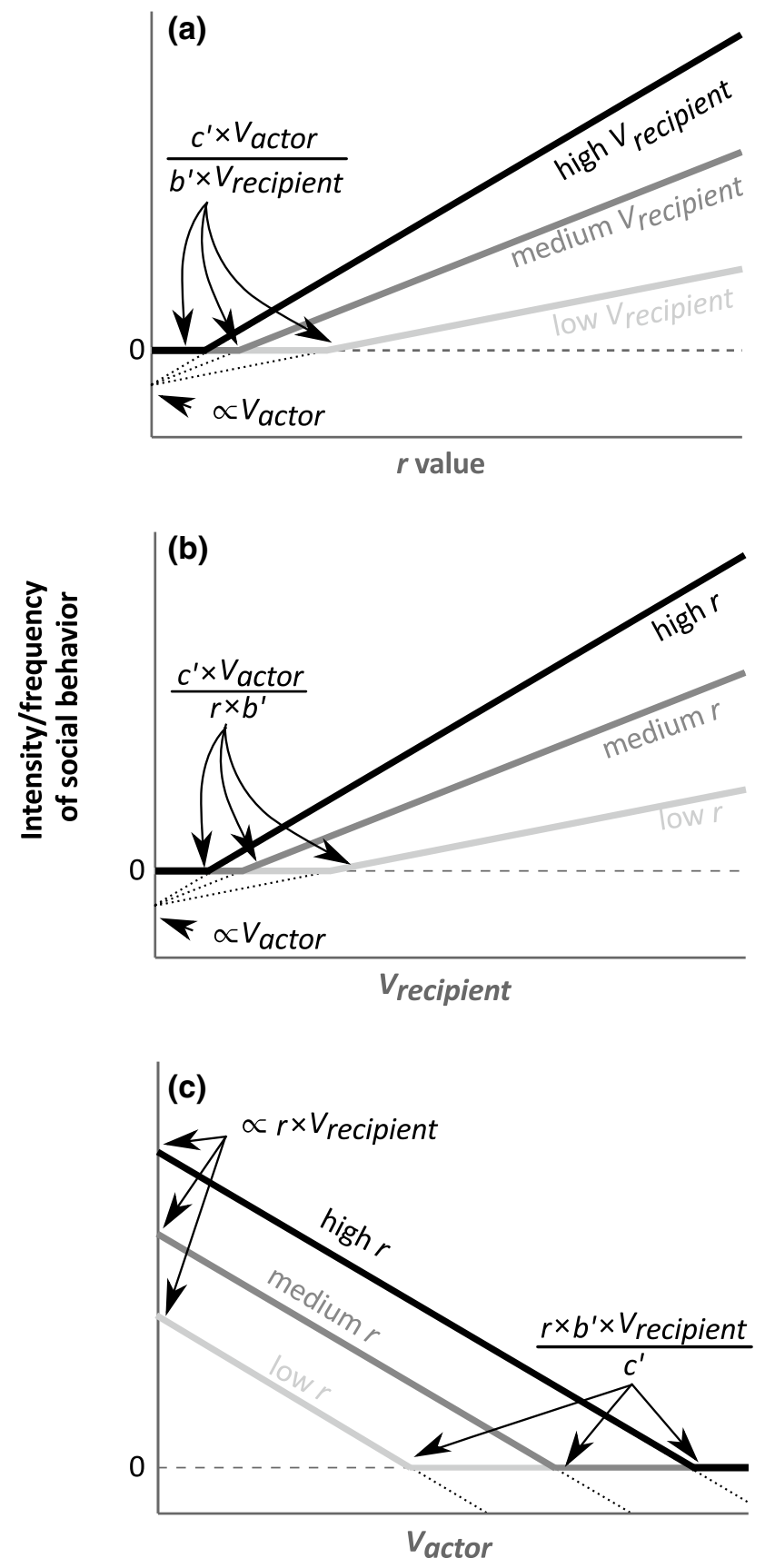

Fig. 4 Prediction of altruistic behavior based on relatedness and reproductive value, all else being equal: intensity of altruistic behavior in relation to a relatedness, $\mathbf{b}$ reproductive value of the recipient, and $\mathbf{c}$ reproductive value of the actors within a given group. Broad lines indicate the predicted intensity/frequency of social behavior. When intensity/frequency of social behavior is predicted to be zero, Hamilton's rule predicts no altruistic behavior. Otherwise, it is predicted that an actor allocates its investment to potential recipients according to relatedness $(r)$ and the expected reproductive value of the recipient at the next time period $\left(V_{\text {recipient }}\right)$. The value of the intercept (and the slope of the line) can change according to the social setting for which the proportional mark $(\alpha)$ is used 


\section{Advantage 2: Distinguishing the kin selection hypothesis from other explanations}

Although many empirical studies support the prediction (see Tables 1,2), there are several alternative explanations of (seemingly) altruistic behaviors other than kin selection, such as reciprocity (West et al. 2007), and these alternative hypotheses may predict a pattern similar to kin selection; that is, high investment in group members with higher reproductive value and relatedness due to the linkage with other variables. For example, suppose that some individuals favor other healthy individuals (e.g., of prime age) who have high reproductive value among relatives due to reciprocity, or for other reasons (e.g., to avoid infection). Relatives may also behave cooperatively because of direct fitness benefits (e.g., Chapais 2001; Silk 2009). Since individual state (e.g., age) often covaries with other characteristics (e.g., experience) of the individual, it is also likely that these characteristics rather than state itself affect the intensity/frequency of social behavior (e.g., the apparently high maternal investment of older mothers, which have low reproductive value, may simply be derived from more successful behavior due to their greater experience; Cameron et al. 2000).

The multiplicative use (i.e., taking the product) of reproductive value and relatedness should improve the situation, as the interaction is not predictable by alternative explanations or confounding factors. In cases in which the approach to quantifying inclusive fitness is impractical (i.e., when $b^{\prime}$ and $c^{\prime}$ cannot be measured), this statistical approach would be the best way to test the kin selection hypothesis for social behavior. Even when reproductive value itself is not available, a linear predictor - if any — can be used as a substitute, broadening the applicability of this statistical tool (e.g., if the focal animal has fixed annual survivorship and fecundity, reproductive value decreases linearly with age after maturation; see also Fig. 1).

Although our prediction, depicted in Fig. 4, is based solely on the kin selection explanation, it is possible to set an additional term to account for the effect of another explanation that is not mutually exclusive to kin selection in its effect on the focal behavior. For example, imagine a case in which the overall intensity/frequency of the focal behavior was determined by both direct and indirect fitness benefits. The intercept value (i.e., the altruistic behavior among nonrelatives) of the linear model can be shifted upward due to the direct fitness effect, while the effects of relatedness and reproductive value can be tested by their interaction and main effects. Therefore, this would not only be the way to distinguish kin selection and other explanations; it would also provide a standard method for evaluating the relative importance of each mechanism.

\section{Caveats}

As previously noted, the predictions above are based on the assumption that the behavior in question has unbiased $b^{\prime}$ and $c^{\prime}$ among group members. When this is not the case, the predictions do not stand, which should be kept in mind. If this caveat is not considered, unrealistic predictions can be made. For example, one might predict that infants should invest more in their mothers rather than vice versa when the infants have lower future reproductive value than their mothers do. This prediction is obviously wrong, as infants have a lower $b^{\prime} / c^{\prime}$ ratio than their mothers, violating the assumption. Another assumption is that the reproductive values of group members should be estimated from a stable population, independent of the given behavior (i.e., the behavior will affect transition rates of the focal individuals but not the reproductive value of each state in the entire population), as in the previous analysis of reproductive value (Table 1). The dynamic change in reproductive value (or nonequilibrium reproductive value)—which depends on the focal behavior-is an alternative approach, at least in theory (e.g., Wild 2011), but its use is impractical in empirical studies. These assumptions might be met in many cases, as in previous studies using reproductive values (Table 1), but should be considered when making predictions using reproductive value.

\section{Conclusion}

We have provided empirical support for the importance of reproductive value in the context of social interaction in mammal societies, although this variable is rarely used in the multiplicative form with relatedness. At present, we are in a better position than ever to apply the product of reproductive value and relatedness because of the accumulating population matrix (e.g., longitudinal research in primates: Kappeler and Watts 2012; see also SalgueroGómez et al. 2016 for a recently established database), more sophisticated statistics to measure reproductive value (e.g., the integral projection model; Rees et al. 2014), and, of course, theoretical development in this study field (e.g., Pen and Weissing 2000; Alizon and Taylor 2008; Johnstone et al. 2012). In a famous episode, J.B.S. Haldane is believed to have said that "he would be prepared to lay down his life for the sake of two brothers or eight cousins" (Davies et al. 2012, p 313; note that this should be "more than" two brothers or eight cousins in a strict sense). From an adaptive perspective, however, one should not forget to weight relatedness using future prospects (i.e., reproductive values) before laying down one's life. 
Acknowledgements This study was financially supported by MEXT (\#25711025 to NK). We are grateful to the members of the laboratory of Sokendai (the Graduate University for Advanced Studies). We also thank Michael Griesser, Fumiaki Nomano, Joe Yuichiro Wakano, and anonymous reviewers for critical comments. The authors have no conflict of interest to declare.

\section{Appendix 1}

\section{See Fig. 5.}

(a)

$\begin{array}{lcccccc} & \begin{array}{c}\text { No. } \\ \text { females }\end{array} & \begin{array}{c}\text { No. } \\ \text { daughters }\end{array} & I_{x} & I_{x} / I_{x-1} & m_{x} & V_{x} \\ \text { Age 0 } & 100 & 0 & 1.00 & - & 0.0 & 1.0 \\ \text { Age 1 } & 50 & 70 & 0.50 & 0.5 & 1.4 & 2.0 \\ \text { Age 2 } & 20 & 30 & 0.20 & 0.4 & 1.5 & 1.5 \\ \text { Age 3 } & 4 & 0 & 0.04 & 0.2 & 0.0 & 0.0\end{array}$

(b)

$\begin{array}{ccccc} & \text { Age 0 } & \text { Age 1 } & \text { Age 2 } & \text { Age 3 } \\ \text { Age 0 } & 0.0 & 1.4 & 1.5 & 0.0 \\ \text { Age 1 } & 0.5 & 0.0 & 0.0 & 0.0 \\ \text { Age 2 } & 0.0 & 0.4 & 0.0 & 0.0 \\ \text { Age 3 } & 0.0 & 0.0 & 0.2 & 0.0\end{array}$

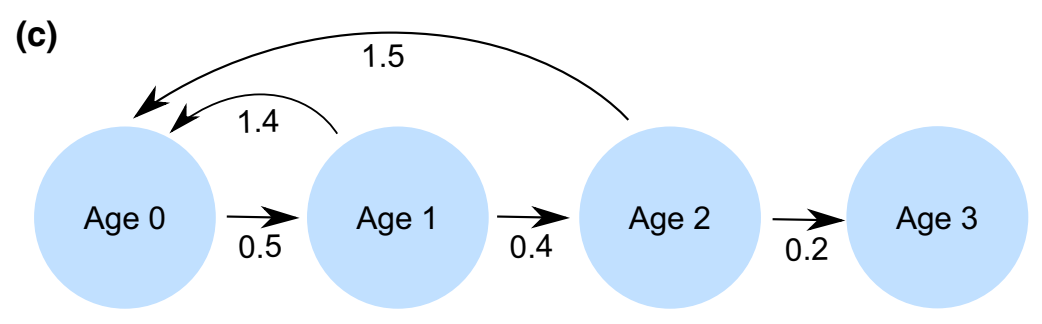

Fig. 5 Population that has been state-structured on the basis of age: a life table with annual survey intervals; b "projection matrix" based on the life table; c life cycle graph, which is mathematically equivalent to the projection matrix. In this example, the population structure is subdivided into ages 0 (infant state), 1 and 2 (reproductive states), and 3 (post-reproductive state). Survivorship and fecundity are denoted as $l_{x}$, (i.e., the probability of surviving to age $x$ ) and $m_{x}$ (i.e., the mean number of female offspring produced per year by a female of age $x$; Fisher 1930), respectively. The reproductive value of age $x\left(V_{x}\right)$ can be calculated via $\exp \left(r_{x}\right) / l_{x}^{*} \sum \exp \left(-r_{t}\right) l_{t} m_{t}$ (which reduces to $\sum l_{t} / l_{x} m_{t}$ in a stable population where $\left.r=0\right)$ in the Leslie matrix or using the dominant left eigenvector in general (note that $r$ is the intrinsic rate of increase per individual). In the life cycle graph c, each state is represented by a node, and arrows between the nodes represent the probabilities of moving from one state to the next (i.e., the transition rate). In the matrix, each movement between states is represented by the value in each cell [i.e., the transition rate from the row to the column in each cell; e.g., the transition rate from "Age 0" (first row) to "Age 1" (second column) is 0.5 , which means that on average $50 \%$ of age 1 individuals survive to age 2; likewise, 1.4 indicates that each age 1 female gives birth to 1.4 daughters on average]. See detailed information on the life table, projection matrix, and life cycle graph given in the literature (e.g., Caswell 1989; Albert and Altmann 2003; Fox 2008)

\section{Appendix 2}

See Fig. 6. 
Fig. 6 A state-structured matrix based on multiple states (age and quality): a projection matrix; b life cycle graph. The time interval is one year. The reproductive values are shown in c. Note that additional information (quality in this case) makes finer predictions than the original Leslie matrix does (e.g., when choosing a recipient, an actor should prefer an age 2 high-quality individual to an age 1 low-quality individual, all else being equal, which is different from the prediction from a Leslie matrix in terms of which age should be preferred; see Fig. 1). Appropriate choice of the state to include is critical when calculating the reproductive values of individuals and predicting the direction of altruistic behavior (a)

\begin{tabular}{|c|c|c|c|c|c|c|c|}
\hline & \multicolumn{2}{|c|}{ Age 0} & \multicolumn{2}{|c|}{ Age 1} & \multicolumn{2}{|c|}{ Age 2} & Age 3 \\
\hline & high & low & high & low & high & low & \\
\hline Age 0 high & 0 & 0 & 1.60 & 0 & 1.70 & 0 & 0 \\
\hline Age 0 low & 0 & 0 & 0 & 1.20 & 0 & 1.30 & 0 \\
\hline Age 1 high & 0.35 & 0.13 & 0 & 0 & 0 & 0 & 0 \\
\hline Age 1 low & 0.15 & 0.37 & 0 & 0 & 0 & 0 & 0 \\
\hline Age 2 high & 0 & 0 & 0.26 & 0.12 & 0 & 0 & 0 \\
\hline Age 2 low & 0 & 0 & 0.14 & 0.28 & 0 & 0 & 0 \\
\hline Age 3 & 0 & 0 & 0 & 0 & 0.22 & 0.18 & 0 \\
\hline
\end{tabular}

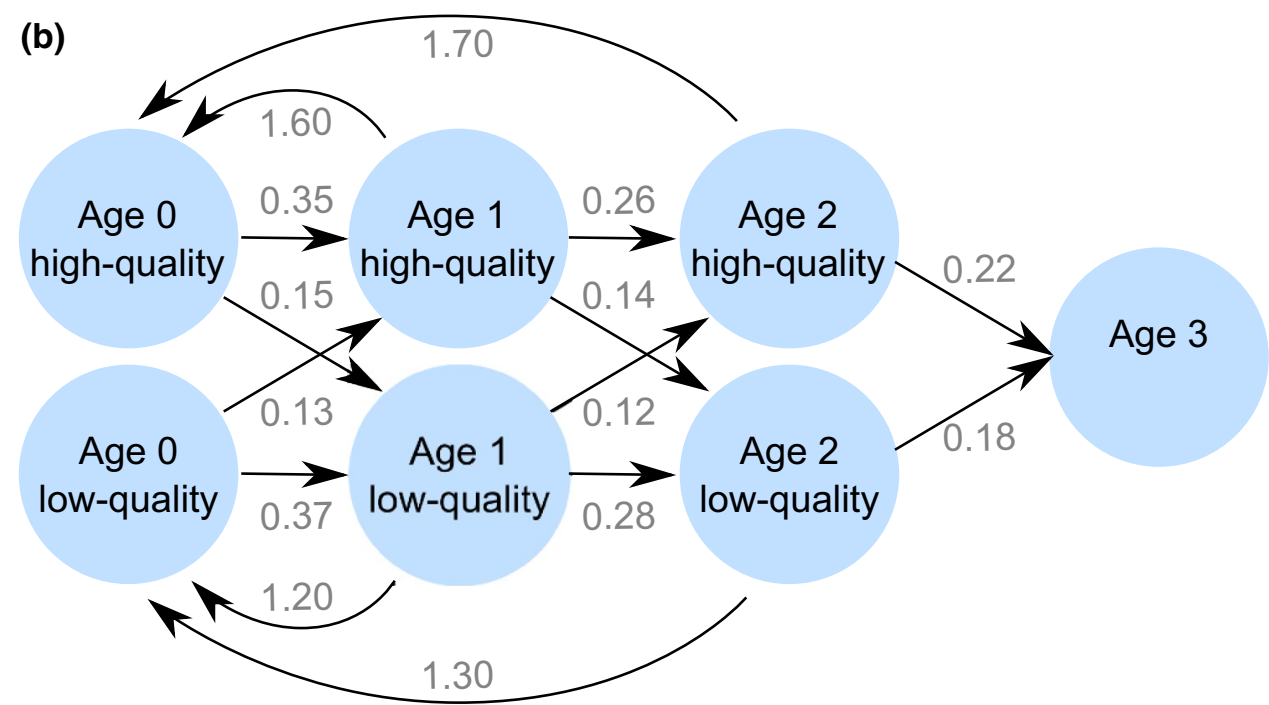

(c)

reproductive value $(V)$

\begin{tabular}{cccc}
\multicolumn{2}{c}{ Age 0} & \multicolumn{2}{c}{ Age 1 } \\
high & low & high & low \\
1.0 & 0.9 & 2.2 & 1.5
\end{tabular}

Age 3

$\begin{array}{ccc}\text { high } & \text { low } & \\ 1.7 & 1.1 & 0.0\end{array}$

\section{Appendix 3}

Age dependency of reproductive value. In an age-structured population, the reproductive value at age $x$ (see Appendix 1) can be rewritten as $V_{x}=m_{x}+l_{x+1} / l_{x}^{*} V_{x+1}$, which can be subdivided into current reproduction $\left(m_{x}\right)$ and residual reproductive value $\left(l_{x+1} / l_{x}^{*} V_{x+1}\right)$. In this equation, it is clear that $V_{x}$ is always smaller than $V_{x+1}$ before maturation (i.e., until $m_{x}>0$ ). The residual reproductive value can decreases with an increase of age after particular age class (which occurs after maturation), and thus, at least when current reproduction $\left(m_{x}\right)$ is a constant or a decreasing function of age, the reproductive value simply decreases with age.

Open Access This article is licensed under a Creative Commons Attribution 4.0 International License, which permits use, sharing, adaptation, distribution and reproduction in any medium or format, as long as you give appropriate credit to the original author(s) and the source, provide a link to the Creative Commons licence, and indicate if changes were made.

The images or other third party material in this article are included in the article's Creative Commons licence, unless indicated otherwise in a credit line to the material. If material is not included in the article's Creative Commons licence and your intended use is not permitted by statutory regulation or exceeds the permitted use, you will need to obtain permission directly from the copyright holder.

To view a copy of this licence, visit http://creativecommons.org/ licenses/by/4.0/.

\section{References}

Albert S, Altmann J (2003) Matrix models for primate life history analysis. In: Kappeler PM, Periera ME (eds) Primate life histories and socioecology. University of Chicago Press, Chicago, pp 66-102

Alizon S, Taylor P (2008) Empty sites can promote altruistic behavior. Evolution 62:1335-1344

Altmann SA (1979) Altruistic behaviour: the fallacy of kin deployment. Anim Behav 27:958-962

Bergman TJ, Beehner JC (2015) Measuring social complexity. Animal Behaviour 103:203-209

Bergmüller R, Johnstone RA, Russell AF, Bshary R (2007) Integrating cooperative breeding into theoretical concepts of cooperation. Behav Process 76:61-72

Borenstein M, Hedges LV, Higgins JPT, Rothstein HR (2009) Introduction to meta-analysis. Wiley, Chichester

Borries C, Sommer V, Srivastava A (1994) Weaving a tight social net: allogrooming in free-ranging female langurs (Presbytis entellus). Int J Primatol 15:421-444 
Bourke AFG (2014) Hamilton's rule and the causes of social evolution. Phil Trans R Soc B 369:20130362

Cameron EZ, Linklater WL, Stafford KJ, Minot EO (2000) Aging and improving reproductive success in horses: declining residual reproductive value or just older and wiser? Behav Ecol Sociobiol 47:243-249

Caswell H (1989) Matrix population models. Sinauer, Sunderland

Chapais B (2001) Primate nepotism: what is the explanatory value of kin selection? Int J Primatol 22:203-220

Clutton-Brock TH (1991) The evolution of parental care. Princeton University Press, Princeton

Combes SL, Altmann J (2001) Status change during adulthood: lifehistory by-product or kin selection based on reproductive value? Proc R Soc Lond B 268:1367-1373

Croft DP, Brent LJN, Franks DW, Cant MA (2015) The evolution of prolonged life after reproduction. Trends Ecol Evol 30:407-416

Davies NB, Krebs JR, West SA (2012) An introduction to behavioural ecology, 4th edn. Wiley-Blackwell, Oxford

de Waal FBM, Tyack PL (2003) Animal social complexity. Harvard University Press, Cambridge

Descamps S, Boutin S, Berteaux D, Gaillard JM (2007) Female red squirrels fit Williams' hypothesis of increasing reproductive effort with increasing age. J Anim Ecol 76:1192-1201

Emlen JM (1973) Ecology: an evolutionary approach. Addison-Wesley, Reading

Fairbanks LA (1988) Vervet monkey grandmothers: interactions with infant grandoffspring. Int J Primatol 9:425-441

Fisher RA (1930) The genetical theory of natural selection. Oxford University Press, Oxford

Fox GA (2008) Discrete analysis (matrix models). eLS. https://doi. org/10.1002/9780470015902.a0003308

Frank SA (1998) Foundations of social evolution. Princeton University Press, Princeton

Grafen A (2006) A theory of Fisher's reproductive value. J Mathemat Biol 53(1):15-60

Hamilton WD (1964) The genetical evolution of social behaviour I \& II. J Theor Biol 7:1-52

Hernández-Pacheco R, Rawlins RG, Kessler MJ, Williams LE, RuizMaldonado TM, González-Martínez J, Ruiz-Lambides AV, Sabat AM (2013) Demographic variability and density-dependent dynamics of a free-ranging rhesus macaque population. Am J Primatol 75:1152-1164

Holekamp KE, Smale L (1993) Ontogeny of dominance in free-living spotted hyenas: juvenile rank relations with other immature individuals. Anim Behav 46:451-466

Houston AI, McNamara JM (1999) Models of adaptive behaviour: an approach based on state. Cambridge University Press, Cambridge

Hrdy SB, Hrdy DB (1976) Hierarchical relations among female Hanuman langurs (Primates: Colobinae, Presbytis entellus). Science 193:913-915

Johnstone RA, Cant MA, Field J (2012) Sex-biased dispersal, haplodiploidy and the evolution of helping in social insects. Proc $\mathrm{R}$ Soc B 279:787-793

Kappeler PM, Watts DP (2012) Long-term field studies of primates. Springer, Berlin

Komdeur J (2012) Sex allocation. In: Royle NJ, Smiseth PT, Kölliker M (eds) The evolution of parental care. Oxford University Press, Oxford, pp 171-188

Koskela E, Juutistenaho P, Mappes T, Oksanen TA (2000) Offspring defense in relation to litter size and age: experiment in the bank vole Clethrionomys glareolus. Evol Ecol 14:99-109

Koskela E, Mappes T, Niskanen T, Rutkowska J (2009) Maternal investment in relation to sex ratio and offspring number in a small mammal: a case for Trivers and Willard theory? J Anim Ecol 78:1007-1014

Kurland JA (1977) Kin selection in the Japanese monkey. Contributions to primatology, vol 12. Karger, Basel, pp 1-145
Kutsukake N (2009) Complexity, dynamics and diversity of sociality in group-living mammals. Ecol Res 24:521-531

Leimar O (1996) Life history analysis of the Trivers and Willard sex ratio problem. Behav Ecol 7:316-325

Lindström J (1999) Early development and fitness in birds and mammals. Trends Ecol Evol 14:343-348

Milinski M (1978) Kin selection and reproductive value. Z Tierpsychol 47:328-329

Montgomerie RD, Weatherhead PJ (1988) Risks and rewards of nest defence by parent birds. Q Rev Biol 63:167-187

Morris DW (1986) Proximate and ultimate controls on life-history variation: the evolution of litter size in white-footed mice (Peromyscus leucopus). Evolution 40:169-181

Nehring V, Boomsma JJ, d'Ettorre P (2012) Wingless virgin queens assume helper roles in Acromyrmex leaf-cutting ants. Curr Biol 22:R671-R673

Oli MK (2003) Hamilton goes empirical: estimation of inclusive fitness from life history. Proc R Soc Lond B 270:307-311

Pen I, Weissing FJ (2000) Towards a unified theory of cooperative breeding. The role of ecology and life history re-examined. Proc R Soc B 267:2411-2418

Pianka ER, Parker WS (1975) Age-specific reproductive tactics. Am Nat 109:453-464

Rees M, Childs DZ, Ellner SP (2014) Building integral projection models: a user's guide. J Anim Ecol 83:528-545

Salguero-Gómez R, Jones OR, Archer CR, Bein C, de Buhr H, Farack C, Gottschalk F, Hartmann A, Henning A, Hoppe G, Römer G, Ruoff T, Sommer V, Wille J, Voigt J, Zeh S, Vieregg D, Buckley YM, Che-Castaldo J, Hodgson D, Scheuerlein A, Caswell H, Vaupel JW (2016) COMADRE: a global data base of animal demography. J Anim Ecol 85:371-384

Schindler S, Gaillard J, Gruning A, Neuhaus P, Traill LW, Tuljapurkar S, Coulson T (2015) Sex-specific demography and generalization of the Trivers-Willard theory. Nature 526:249-252

Schulman SR, Chapais B (1980) Reproductive value and rank relations among macaque sisters. Am Natl 115:580-593

Schwanz LE (2008a) Chronic parasitic infection alters reproductive output in deer mice. Behav Ecol Sociobiol 62:1351-1358

Schwanz LE (2008b) Persistent effects of maternal parasitic infection on offspring fitness: implications for adaptive reproductive strategies when parasitized. Funct Ecol 22:691-698

Schwartz OA, Armitage KB, Van Vuren D (1998) A 32-year demography of yellow-bellied marmots (Marmota flaviventris). J Zool 264:337-346

Silk JB (2009) Nepotistic cooperation in non-human primate groups. Philos Trans R Soc Lond B 364:3243-3254

Smith JE (2014) Hamilton's legacy: kinship, cooperation and social tolerance in mammalian groups. Anim Behav 92:291-304

Taylor PD (1990) Allele-frequency change in a class-structured population. Am Natl 135:95-106

Taylor PD, Frank S (1996) How to make a kin selection model. J Theor Biol 180:27-37

Trivers RL, Willard DE (1973) Natural selection of parental ability to vary the sex ratio of offspring. Science 179:90-92

Van Horn RC, Buchan JC, Altmann J, Alberts SC (2007) Divided destinies: group choice by female savannah baboons during social group fission. Behav Ecol Sociobiol 61:1823-1867

West SA, Grrifin AS, Gardner A (2007) Evolutionary explanations for cooperation. Curr Biol 17:R661-R672

Wild G (2011) Direct fitness for dynamic kin selection. J Evol Biol 24:1598-1610

Publisher's Note Springer Nature remains neutral with regard to jurisdictional claims in published maps and institutional affiliations. 